\title{
Investigating Elastic Relaxation Effects on the Optical Properties of Functionalised Calcium Carbonate Compacts using Optics-Based Heckel Analysis
}

Prince Bawuah ${ }^{\mathrm{a}^{*}}$, Anssi-Pekka Karttunen ${ }^{\mathrm{a}}$, Daniel Markl ${ }^{\mathrm{b}}$, Cathy Ridgwayc, Ossi Korhonen ${ }^{\mathrm{a}}$, Patrick Gane ${ }^{\mathrm{c}, \mathrm{d}}$, J. Axel Zeitler ${ }^{\mathrm{e}}$, Jarkko Ketolainen ${ }^{\mathrm{a}}$, Kai-Erik Peiponen ${ }^{\mathrm{f}}$

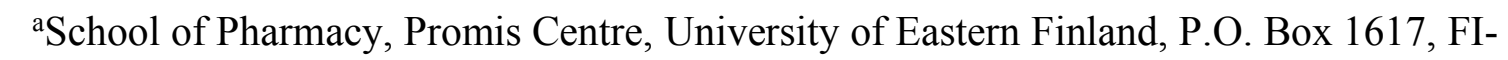
70211, Kuopio, Finland

${ }^{\mathrm{b}}$ Strathclyde Institute of Pharmacy and Biomedical Sciences, University of Strathclyde, 161 Cathedral Street, G4 0RE Glasgow, UK

'Omya International AG, CH-4665 Oftringen, Switzerland

dAalto University, Chemical Engineering, Bioproducts and Biosystems, FI-00076 Aalto, Helsinki, Finland

e Department of Chemical Engineering and Biotechnology, University of Cambridge, Philippa Fawcett Drive,CB3 0AS Cambridge, United Kingdom

fInstitute of Photonics, University of Eastern Finland, P.O. Box 111, FI-80101 Joensuu, Finland

Corresponding author: Prince Bawuah

Electronic address: prince.bawuah@uef.fi

Phone: +358504120591

Address: School of Pharmacy, Promis Centre, University of Eastern Finland, P.O. Box 1617, FI-70211, Kuopio, Finland 


\section{Abstract}

Heckel analysis is a widely used method for the characterisation of the compression behaviour of pharmaceutical samples during the preparation of solid dosage formulations. The present study introduces an optical version of the Heckel equation that is based on combination of the conventional Heckel equation together with the linear relationship defined between the effective terahertz $(\mathrm{THz})$ refractive index and the porosity of pharmaceutical tablets. The proposed optical Heckel equation allows us to firstly, calculate the zero-porosity refractive index, and secondly, predict the in-die development of the effective refractive index as a function of the compressive pressure during tablet compression. This was demonstrated for five batches of highly porous functionalised calcium carbonate (FCC) excipient compacts. The close match observed between the estimated in-die effective refractive index and the measured/out-of-die effective $\mathrm{THz}$ refractive index confirms the validity of the proposed form of the equation. By comparing the measured and estimated in-die tablet properties, a clear change in the porosity and hence, the effective refractive index, due to after-compression elastic relaxation of the FCC compacts, has been observed. We have, therefore, proposed a THz-based compaction setup that will permit in-line monitoring of processes during tablet compression. We envisage that this new approach in tracking powder properties introduced in this preliminary study will lead to the onset of further extensive and detailed future studies.

Keywords: Heckel law; pharmaceutical tablet; elastic relaxation; porosity; terahertz refractive index

\section{Introduction}

The numerous advantages that come with the use of pharmaceutical tablets have led to significant investments by the pharmaceutical industry into the study of powder compaction as well as controlling the quality of the finished products. Typical pharmaceutical tablets are composed of several excipient and active pharmaceutical ingredient (API) particles that undergo a vast complexity of processes during compression. The initially loose powder bed undergoes dramatic changes as a function of the relative density due to the force of compaction applied by the tablet punch. These changes include particle rearrangements, elastic and plastic deformation as well as brittle fracture of particles depending on their mechanical properties. Moreover, porosity, distribution of internal stress and density (Kawakita and Lüdde, 1971; Ryshkewitch, 1953) as well as the crystal habit of a drug (Rasenack and Müller, 2002) affect the tableting behavior and the quality properties of the finished tablet. 
Despite sustained research into powder compaction there are still open questions regarding the complex nature of tableting processes. It is still challenging to successfully predict the properties of the end-tablet product even when all the compositions of the powder mixture are exactly known. Over the past decades a number of experimental techniques and a wide variety of compaction models (Çelik, 1992; Çelik and Marshall, 1989; Krycer et al., 1982; Paronen, 1986; Salleh et al., 2015; Sun and Grant, 2001) have been developed and utilised to characterise compression behaviour, i.e. compressibility, compactibility, and pressure susceptibility of pharmaceutical powders. These models are mostly empirical and define criteria to guide the rational selection of suitable excipients to meet the desired properties of the dosage form. However, many of these models are valid within a restricted range of compaction force and can only describe the compaction process of specific pharmaceutical materials (Çelik, 1992; Kolařík, 1994; Van Veen et al., 2004; Wu et al., 2005).

The most widespread models are described in terms of the Heckel equation (Heckel, 1961a, 1961b), the Kawakita equation (Lüdde and Kawakita, 1966), the Drucker-Prager-Cap model (Han et al., 2008), the modified Heckel equation by Kuentz and Leuenberger (Kuentz and Leuenberger, 1999) and the approach according to Cooper and Eaton (Cooper and Eaton, 1962). The Heckel model, which is by far the most popular in the field of powder compression, is of special interest for the present study. Although several authors have highlighted a number of limitations (Rue and REES, 1978) and have proposed modified versions of the Heckel model (Stirnimann et al., 2014), its chief merit is its simplicity together with the readily available reference dataset for different kinds of pharmaceutical materials. This makes the analysis and comparison of different pharmaceutical materials more convenient when the Heckel equation is adopted.

Based on the inevitable need for an analytical model during the preparation of solid dosage formulations, the present study considers the Heckel equation but introduces an optical-related version, which is henceforth referred to as "optical Heckel". The proposed optical Heckel concept originates from the linear correlation observed between the terahertz (THz) based bulk refractive index (effective refractive index) and the bulk porosity of pharmaceutical tablets (Bawuah et al., 2016b; Markl et al., 2018a). With terahertz time-domain spectroscopy (THzTDS), the effective refractive index, which is a function of the total porosity of a given tablet, can be measured in a nondestructive and contactless fashion within seconds. Based on the unique advantages associated with optical/THz based measurement techniques, we believe that this new optical Heckel equation can serve as a complementary model for rendering 
comprehensive insight into the processes and compaction mechanisms of pharmaceutical powders.

Pharmaceutical industry is currently under transition from traditional batch manufacture to continuous manufacture (Lee et al., 2015; Nasr et al., 2017). One improvement that is usually coupled with continuous manufacture is continuous process monitoring using Process Analytical Technology (PAT) tools. A key to successful implementation of real-time release (RTR) is the ability to monitor processes continuously based on real-time analysis and control of the manufacturing process. Since the compaction process is one of the key operations in a continuous manufacturing plant, it is particularly important to control the physical and chemical properties of tablets. RTR testing is only possible on the basis of a firm understanding of the process and the relationship between process parameters, material attributes and product attributes (European Medicines Agency, 2012). A robust physical model that is sensitive enough and intuitive to use is a key requirement to design and control tablets with required/specific out-of-die properties. This is possible by utilising observed relations between THz-TDS and important tablet parameters, as we have reported in our previous THz-TDS based experiments (Bawuah et al., 2016a, 2016b, 2014a, 2014b, Chakraborty et al., 2017, 2016, Markl et al., 2017a, 2017b).

Here, we verify the validity of the proposed optical Heckel method using a two-phase pharmaceutical compact consisting of air-filled pores and a solid material, in the form of a recently developed excipient, functionalised calcium carbonated (FCC), chosen due its complex porous particle behaviour unlike that of other commonly used solid excipients. This excipient presents a more challenging case study for applying conventional modelling approaches.

The present study also investigates the existence of elastic relaxation of the FCC compacts after compression based on their in-die and out-of-die porosity as well as height values. To further ascertain the influence of the elastic relaxation on the optical properties of tablets during and after compression, this study estimates the in-die effective refractive index based on the proposed optical Heckel method and compares to the measured counterpart. Elastic relaxation during (Anuar and Briscoe, 2009) and after (Baily and York, 1976) ejection has major influence on, especially, the mechanical and microstructural properties of the finished tablets. We believe that by successfully introducing these optical methods through a carefully engineered 
compaction setup, it is possible to realise in-line quality control of each tablet during and after compression.

\section{Theory}

The conventional Heckel equation (Heckel, 1961a, 1961b) describes the relationship between the logarithmic inverse of the porosity, $f=1-\delta$ with $\delta$ as the relative density, and the applied compressive pressure, $p$. The Heckel equation was derived based on the assumption that the in-die densification of the bulk powder obeys first order kinetics as,

$\ln \left(\frac{1}{f}\right)=-\ln f=K p+A$

where $K$ is a constant of proportionality describing the development of a log-linear response of the structure to the application of pressure, i.e. the Heckel slope, and $A$ is an intercept constant describing densification by particle movement and rearrangement. The inverse of $K$ is the mean yield pressure and it represents the limit of plastic deformation of materials or the resistance of a material to deformation (Hersey and Rees, 1971).

The measurement of the refractive index of tablets via THz-TDS has been studied extensively using both the frequency-domain (Bawuah et al., 2016b; Markl et al., 2017b) and time-domain analytical approaches (Bawuah et al., 2016b, 2014a; Markl et al., 2017b). The bulk THz refractive index measured for a given tablet is also referred to as the effective refractive index $\left(n_{\text {eff }}\right)$ due to the multicomponent nature of a typical pharmaceutical tablet (Bawuah et al., 2014a). Based on empirical evidence, a linear correlation between the effective refractive index and the porosity of pharmaceutical tablets composed of different materials and covering a wide range of porosities was observed (Markl et al., 2018a).

$n_{\mathrm{eff}}(f)=n(0)+(1-n(0)) f$

where $n(0)$ represents the zero-porosity refractive index, i.e. the inherent refractive index of the the solid material constituent in the absence of any imperfections or porosity. During data acquisition, nitrogen gas was used to purge the sample compartment to reduce the effect of water vapor on the terahertz transmission measurement and hence, the unity in Eq. (2) represents the refractive index of air/nitrogen gas $\left(n_{\text {air }}=1\right)$. Eq. (2) is called the zero-porosity approximation (ZPA) method, which is a complementary method, vis-a-vis effective medium theory (EMT), for the estimation of the porosity from known $n_{\text {eff }}$ of a given tablet (Markl et al., 2017b). However, the estimation of porosity of pharmaceutical tablets using Eq. (2) is outside the scope of this study. In the present study, we intend rather to monitor the in-die development 
of $n_{\text {eff }}$ during the compression process using Eq. (2). With a compaction simulator, it is possible to measure the in-die porosity based on in-die densification of the bulk powder, i.e. change in the relative density with respect to increasing compressive force. Equation (2), therefore, serves as the basis for the derivation of the proposed optical counterpart of the Heckel law. From Eqs. (1) and (2) we can solve the following expression for the pressure dependent effective refractive index as follows:

$n_{\mathrm{eff}}(p ; f)=n(0)-(n(0)-1) e^{-(K p+A)}$

From Eq. (3), we claim that $n_{\text {eff }}$ is directly proportional to the result of the Heckel law, and hence it can be used as an alternative or a complementary quantity to $\ln (1 / f)$. Moreover, based on the direct use of Eq. (2), i.e. in the derivation of Eq. (3), as well as its applicability for different types of pharmaceutical powders (Markl et al., 2018a), we propose that the optical Heckel model can serve as a complemetary general model for characterising the compaction beviour of different types of pharmaceutical powders. The compression process can be treated as an adiabatic thermodynamic process, where all the thermodynamic variables, such as, volume, pressure and absolute temperature $(T)$ are subject to change. In a study conducted by Wurster and Buckner (Wurster and Buckner, 2012), compaction-induced thermodynamic changes during the compression of anhydrous lactose, a common pharmaceutical excipient, has been characterised.

To tackle the mechanisms involved in the compression process of the powder bed rigorously, one should take into consideration the effect of temperature and pressure on the $n_{\text {eff }}$ (Wax and Cleek, 1973). However, taking into consideration the temperature dependent $n_{\text {eff }}$ will yield a quite challenging situation due to the porous nature of a tablet, which comprises both solid medium and air. In principle, the solid skeleton could be treated by invoking the Mie-Grüneisen model and considering air as an ideal gas similar to dealing with the state equation of highly porous materials with closed and isolated pores (Belkheeva, 2015). Even with this assumption, it is still quite cumbersome to derive the equation of state for pharmaceutical compacts due to their complex nature, i.e. a typical tablet consists of API and various excipient particles as well as both isolated and connected pores. Based on the abovementioned difficulties, this study employs a simple expression as given in Eq. (3) to powder compression. In parallel, we wish to remark that it is possible, in principle, to gain experimental information on both the temperature and pressure dependence of effective refractive index (Wax and Cleek, 1973). Nonetheless, the ZPA refractive index, $n(0)$, used in the calculations of this study is obtained 
at normal atmospheric pressure and at room temperature. Obviously, for rigorous analysis of the in-die data, the zero porosity refractive index $n(0)$ should be replaced by high pressure and absolute temperature dependent zero porosity refractive index, $n(0, p, T)$, which corresponds to the thermodynamic state of the compact inside the die. However, the thermodynamically dependent refractive indices of the FCC compacts are unknown. Therefore, the present study utilises the estimated zero porosity refractive index, $n(0)$.

\section{Materials and Methods}

\subsection{Materials}

The training set of powder compacts was prepared from FCC (Omyapharm ${ }^{\circledR}$; Omya International AG, Oftringen, Switzerland). The FCC powder is composed of core-shell structured particles in which agglomerated ultrafine calcium carbonate particulates are surrounded by lamellae of hydroxyapatite, thus forming a dual porosity compact. A detailed discussion on the formation of FCC (a highly porous plate-like, nanometre thick lamellar structure of high surface area) has been reported previously (Markl et al., 2017b). A typical pharmaceutical FCC particle consists of a calcium carbonate/hydroxyapatite ratio of $15 \%$ $20 \%$ calcium carbonate to $80 \%$ - $85 \%$ hydroxyapatite.

\subsection{Tableting}

With a compaction simulator (PuuMan Ltd, Kuopio, Finland), the FCC powder was directly compressed into flamt-faced, cylindrical tablets with target height and diameter of $1.5 \mathrm{~mm}$ and $10 \mathrm{~mm}$, respectively. A range of final compaction levels were achieved by varying the mass of material in the tablet, resulting in tablets that spanned a wide range of porosities. The highly porous nature of the FCC particles permitted the manufacturing of five batches of tablets with increasing porosity starting from a targeted total porosity of $45 \%$ rising to $65 \%$ in $5 \%$ increments (Table 1). Each batch consisted of 15 tablets.

During the compaction process, both the upper and lower punch forces as well as upper punch displacement were logged. Figs. 1(a) and (b) show both the compressive force and displacement-time profiles for each batch of tablets. The in-die tablet thickness/height at any compressive pressure (Fig. 1(c)) was tracked as the difference between the lower punch displacement and the upper punch displacement. It is worth mentioning that during the calculation of the punch displacement, deformation of the tooling was taken into consideration.

The in-die tablet density was then calculated from the weight and thickness of the tablet at a given compressive pressure. Given the true density of the material in conjuction with the known 
in-die tablet density, the relative density, and, hence, the in-die porosity, were extracted. We wish to mention that the in-die paramater extraction approach does not take into account the elastic relaxation that occurs after the compaction step. The elastic relaxation causes an increase of the tablet height and porosity, which in turn influences other material parameters extracted from the fitted Heckel plots (Çelik, 1992). In a study conducted by Celik and Marshall (Çelik and Marshall, 1989), it was observed that the differences in the tablet dimensions due to the use of the in-die and out-of-die methods significantly influenced the final Heckel plots. Nonetheless, due to the ease of data collection as well as measurement speed, the in-die approach is still widely used.

\subsection{Methods}

Terahertz Time-Domain Spectroscopy (THz-TDS): Terahertz time-domain measurements were acquired using a Terapulse 4000 spectrometer (Teraview Ltd., Cambridge, UK) in transmission mode. The transmission chamber was purged with dry nitrogen gas throughout the measurement and the noise was reduced by co-averaging 60 measurements. Each timedomain waveform covered a range of $150 \mathrm{ps}$ using a resolution of $0.1 \mathrm{ps}$, and the total measurement time of the co-averaged waveforms was $1.5 \mathrm{~min}$. The effective refractive index,

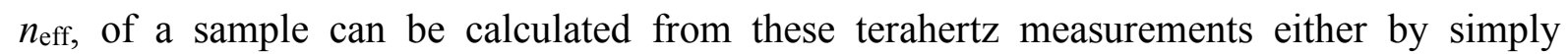
comparing a reference waveform from a known material, e.g. an empty chamber that is purged with dry nitrogen gas $\left(n_{\text {air }}=1\right)$, or by considering the fraction of components of known refractive index in the tablet under a range of porosities and extrapolating to zero porosity to provide the reference for scaling. Since the particle size of FCC $(12.1 \mu \mathrm{m})$ is significantly smaller than the wavelength, $\lambda=300 \mu \mathrm{m}$, at which we evaluated the refractive index, the effect of scattering is negligible. Therefore, a reduction in particle size during the compaction does not influence the refractive index measurements in terms of scattering. In other words, any dynamic change of sub wavelength particle size is lost.

\section{Results and discussion}

In this study, both in-die and out-of-die Heckel analysis based on the conventional Heckel equation and the proposed optical Heckel equation have been performed using compression data of FCC compacts.

\subsection{Conventional Heckel analysis}

For the sake of comparison, we have performed both in-die and out-of-die conventional Heckel plots as shown in Fig. 2. In each case, the compression characteristics of FCC were determined 
by estimating the slope $(K)$ and the intercept $(A)$ from the Heckel plots (Eq. (1)). The linear portion of the plot was carefully selected to obtained a similar range of compressive pressure for both the in-die and out-of-die data. To meet this condition, only the set B01 was used for the in-die log-linear extrapolation analysis. The slope was obtained by fitting a straight line, whose correlation coefficients $\left(R^{2}\right)$ was not less than 0.99 , through data points within the compressive pressure range of around 250 - $370 \mathrm{MPa}$.

Naturally, one would have expected to observe an overlap of the different curves from the indie Heckel plots for the various batches, since the batches were compressed with the same material (FCC). The shift observed in the Heckel plots means that even though the batches contain the same material, they will have different compression properties, e.g. different slopes and, hence, different yield pressures. The inconsistencies observed emphasise the fact that the conventional Heckel law does not take into consideration the pressure susceptibility, which is quite predominant at high relative densities and pressures (Kuentz and Leuenberger, 1999). At low pressures the compression process is dominated by particle rearrangements. In effect, tablets formed at low pressures are essentially particle agglomerates rather than homogeneously dispersed holes in a solid matrix, as rightly stated by Kuentz and Leuenberger (Kuentz and Leuenberger, 1999). Hence, it is not surprising to observe significant differences in the physical behaviour of tablets created at low compressive pressures - in other words tablets with low relative density or high porosity, as demontrasted in the present study (see Fig. 2). Kuentz and Leuenberger (Kuentz and Leuenberger, 1999), therefore, introduced a threshold for the porosity or the relative density at which rigidity starts to evolve. That is, a pressure susceptibility parameter should be taken into consideration for compacts with porosities below the threshold porosity. In this study, the focus was not to adopt the modified Heckel law according to Kuentz and Leuenberger (Kuentz and Leuenberger, 1999), hence, the threshold porosity for the FCC tablets was not estimated. The model in (Kuentz and Leuenberger, 1999) was invoked just to explain the behaviour of the curves in Fig. 2. Nonetheless, such modification can easily be implemented later on if needed.

Finally, the differences in values recorded for both $K$ and $A$ in the in-die and out-of-die analyses are due to the relatively low porosities (see Table 2) used in the in-die analysis compared to the relative high porosity values for the out-of-die analysis. The increase in porosity values during the out-of-die analysis is a result of elastic relaxation of the tablets after compression. To buttress our claim of the possible existence of elastic relaxation of the tablets after compression, we tabulate and compare both the in-die and out-of-die tablet thicknesses in Table 
2. The percentage relative difference, $\delta J$, between the in-die and out-of-die values of the porosity and height/thickness of the tablets is given by

$\delta J=\frac{\left|J_{\text {out }}-J_{\text {in }}\right|}{\left|J_{\text {in }}\right|} \times 100$

where $J$ is the tablet parameter, i.e. porosity or height, under consideration, and $J_{\text {out }}$ as well as $J_{\text {in }}$ are the respective out-of-die and in-die values of the parameter. The results obtained (Table 2) indicate relatively high porosity changes, i.e. significant elastic relaxation after compression, for the batch formed under high compressive pressure. Similarly, the relative change in height, as a result of elastic relaxation after compression, is clearly observed for all the batches (Table 2).

\subsection{Optical Heckel analysis}

Similar analysis, as in the case of the conventional Heckel plots (Fig. 2), were performed using the optical Heckel concept as given by Eq. (3). In other words, it is possible to predict, using Eqs. (2) and (3), the development of the effective refractive index during the compressive process of a pharmaceutical compact.

Two major analytical steps were taken during the optical Heckel analyses. Firstly, the $K$ and $A$ values, obtained from the in-die conventional Heckel analysis (Fig. 2), were used in addition to the measured $\mathrm{THz}$ effective refractive index and the maximum compressive pressure, to calculate the in-die zero-porosity refractive index, $n(0)=n_{\text {in }}(0)$ given in Table 3 . The relative change between the estimated $n_{\text {in }}(0)$ with respect to the already known out-of-die zero-porosity refractive index, i.e. $n(0)=n_{\text {out }}(0)=2.97$ for FCC powder (Markl et al., 2017b), was calculated (Eq. (4)) and tabulated (Table 3 ). The predicted $n_{\text {in }}(0)$ values, as listed in Table 3 , are in a good agreement with the $n_{\text {out }}(0)=2.97$.

Secondly, by using the average value of the $n_{\text {in }}(0)$, i.e. $n_{\text {in }}(0)=3.088$ with $\delta n(0)=3.97 \%$, in combination with the known values of $K, A$ and the in-die compressive pressure, the in-die effective refractive index, $n_{\text {eff.in }}(p ; f)$, defined by Eq. (3), was calculated and compared to the out-of-die effective refractive index, $n_{\text {eff.out }}$ (see Fig. 3). Also, the in-die effective refractive index at maximum compressive pressure, $n_{\mathrm{eff}, \mathrm{in}}\left(p_{\max }\right)$, is compared to the measured (out-of-die) $\mathrm{THz}$ effective refractive index, $n_{\text {eff.out }}$ (Table 3). The black dots in Fig. 3 indicate the $n_{\text {eff.in. }}$.

A close match is observed between the in-die and the out-of-die effective refractive index as shown in Fig. 3 where the calculated in-die effective refractive index data nicely fits the data of the out-of-die effective refractive index. The calculated low relative change, $\delta n_{\mathrm{eff}}$, of the 
effective refractive index (Table 3) also attests to the observed close match between the in-die and the out-of-die effective refractive index, which proves the accuracy of the estimated ZPA $n(0)$ using this newly proprosed optical Heckel method. In other words, the proposed optical Heckel method can serve as a complementary method to the ZPA method for accurate prediction of the zero-porosity refractive index of tablets. However, the relative low differences observed between the values of the calculated in-die effective refractive index, $n_{\text {eff.in, }}$, and the measured/out-of-die effective refractive index ( $\left.n_{\text {eff.out }}\right)$, might be attributed to the presents of elastic relaxation of the tablets after compression. Additionally, the use of the simple ZPA $n(0)$ in the calculations without taking into consideration the effect of the relatively high in-die temperature and pressure during compression, as already mentioned in section 2, can be a contributing factor to the observed relative change in the effective and intrinsic refractive indices. Finally, differences in the moisture content of the samples, as well as experimental errors during the in-die and out-of-die measurements, can significantly contribute to the observed discrepancies in the refractive indices.

The above promising experimental observation in addition to the zero offset in the optical Heckel plots (Fig. 3) suggest the validity of the linear relation between $n_{\text {eff }}$ and $f$ as defined in Eq. (2) and utilised in Eq. (3). In other words, the purely experimental data gives support to the theoretical models given in Eqs. (2) and (3). The effective refractive index is a basic optical property that can be measured easily by terahertz time-domain technology (Bawuah et al., 2016a, 2016b, 2014a, 2014b, Chakraborty et al., 2017, 2016, Markl et al., 2017a, 2017b). This makes terahertz spectroscopy a promising PAT tool for in-line applications in the pharmaceutical industry.

Fig. 4 summarises and compares all the estimated relative changes, i.e. porosity, height, effective refractive index, and intrinsic refractive index, that might be caused by the aftercompression elastic relaxation of the FCC compacts. There is clearly a change in the porosity as well as effective refractive index due to elastic relaxation. However, the observed relative change in the porosity is not directly correlated to that of the effective refractive index, which buttresses our previous speculation that, aside porosity; thermodynamic parameters, e.g. temperature, have significant influence on the effective refractive index of pharmaceutical tablets, especially during compression. Similarly, the change in the porosity is not directly correlated to the tablet thickness change (Fig. 4). The change in the effective refractive index and porosity clearly indicates the influence of elastic relaxation on the pore structure. This will in turn impact the liquid uptake rate that is part of the disintegration process of pharmaceutical 
tablets (Markl et al., 2018b). This post-compaction variation of the pore structure will also directly affect the dissolution performance. It is thus of great importance to understand the elastic relaxation effect in the light of quality control - dissolution studies may yield different results when conducted right after compaction compared to them performed several days or weeks after manufacturing. Moreover, the elastic relaxation is a critical mechanism in continuous manufacturing as the change in tablet volume may cause cracks in the subsequently applied coating, which may render the coating function, e.g. improving the appearance, masking an odour, modifying the release characteristics, useless.

The close match between the values of the calculated and measured refractive index is an indication of the possibility to conduct optical Heckel analysis using $\mathrm{THz}$ technology. By engineering a compaction setup coupled with terahertz pulse imaging (TPI), it would be possible, in principle, to measure the in-die refractive index at a given compressive force as illustrated in the schematic diagram shown in Fig. 5. With the known in-die refractive index, we can extract the values of $K$ and $A$ from a $\ln \left(n_{\text {eff }}(p ; f)\right)$ versus compressive pressure plots (Eq. (3)). One advantage of the proposed optical Heckel analysis over the conventional Heckel analysis is the use of a basic optical property, i.e. refractive index that can be measured directly by $\mathrm{THz}$ means. At present, there are no good techniques available to measure porosity in-die (except maybe the use of in situ ultrasound approach), hence, the porosity used in the conventional Heckel plots is mostly calculated from the bulk and true densities of a given powder material. However, accurate measurements of the true density of pharmaceutical powder, especially hydrates, by using helium pycnometry, has raised major concern (Sun, 2004). It is important to mention that, currently, the use of the THz-TDS in the detection of the effective refractive index of pharmaceutical tablets is limited to out-of-die method only. Nevertheless, the relatively good match between the calculated (in-die) and measured (out-ofdie) refractive index data shows the power of the proposed optical Heckel law to estimate $K$ and $A$ accurately from a non-contact measurement of $\mathrm{THz}$ pulse under standard condition (atmospheric pressure). Finally, aside from confirming the validity of the linear relation (Eq. (2)), the close similarity of the calculated $\left(n_{\text {eff.in }}\right)$ and measured $\left(n_{\text {eff.out }}\right)$ data also means that both in-die and out-of-die porosity of tablets can now be estimated from their $\mathrm{THz}$ refractive index data without using the current not ideal true density approach.

\section{Conclusions}

This study has successfully introduced and practically demonstrated the newly proposed optical Heckel concepts for fast, non-invasive and accurate characterisation of the compression 
behaviour of FCC compacts. As a case study, five batches of compressed FCC compacts with different porosity levels were used in the study. The proposed optical Heckel equation was derived based on modified mathematical models formulated as a result of our previous $\mathrm{THz}$ TDS experiments. Close correlations were observed between the in-die and out-of-die optical Heckel analyses without any significant offsets. The close match between the values of the predicted effective $\mathrm{THz}$ refractive index, i.e. based on the optical Heckel equation, and the measured $\mathrm{THz}$ refractive index of the FCC compacts has shown the feasibility of replacing the porosity with the effective $\mathrm{THz}$ refractive index during Heckel analysis. We will work on extending the optical Heckel concept to other popular models such as Leuenberger, and Kawakita using different pharmaceutical materials and biconvex tablets.

The present study has also investigated the existence of elastic relaxation of FCC compacts after compression based on the differences observed in both their in-die and out-of-die porosity as well as height values. Furthermore, the study has highlighted the effect of after-compression elastic relaxation on the optical properties, i.e. effective refractive index, of FCC compacts using the newly proposed optical Heckel method. Based on a comparison between the calculated in-die effective refractive index and the measured out-of-die effective refractive index, a clear change in the effective refractive index of the FCC compacts has been observed. The relative change in the effective refractive index is attributed partly to elastic relaxation and partly to thermodynamic effects as well as possible experimental errors.

Finally, the proposed optical Heckel method can serve as a complementary method to the ZPA method for accurate prediction of the zero-porosity refractive index of pharmaceutical tablets. Hence, as an initiator of future study, the present work proposes a compaction setup that will allow in-line process monitoring using $\mathrm{THz}$ technology.

\section{References}

Anuar, M.S., Briscoe, B.J., 2009. The elastic relaxation of starch tablets during ejection. Powder Technol. 195, 96-104. https://doi.org/10.1016/j.powtec.2009.05.019

Baily, E.D., York, P., 1976. An apparatus for the study of strain recovery in compacts. J. Mater. Sci. 11, 1470-1474. https://doi.org/10.1007/BF00540880

Bawuah, P., Chakraborty, M., Ervasti, T., Zeitler, J.A., Ketolainen, J., Gane, P.A.C., Peiponen, K.-E., 2016a. A structure parameter for porous media obtained with the aid of Wiener bounds for effective permittivity and terahertz time-delay measurement. Int. J. Pharm. 506, 87-92. https://doi.org/10.1016/j.ijpharm.2016.04.026 
Bawuah, P., Pierotic Mendia, A., Silfsten, P., Pääkkönen, P., Ervasti, T., Ketolainen, J., Zeitler, J.A., Peiponen, K.-E., 2014a. Detection of porosity of pharmaceutical compacts by terahertz radiation transmission and light reflection measurement techniques. Int. J. Pharm. 465, 70-76. https://doi.org/10.1016/j.ijpharm.2014.02.011

Bawuah, P., Silfsten, P., Ervasti, T., Ketolainen, J., Zeitler, J.A., Peiponen, K.-E., 2014b. Non-contact weight measurement of flat-faced pharmaceutical tablets using terahertz transmission pulse delay measurements. Int. J. Pharm. 476, 16-22. https://doi.org/10.1016/j.ijpharm.2014.09.027

Bawuah, P., Tan, N., Tweneboah, S.N.A., Ervasti, T., Axel Zeitler, J., Ketolainen, J., Peiponen, K.-E., 2016b. Terahertz study on porosity and mass fraction of active pharmaceutical ingredient of pharmaceutical tablets. Eur. J. Pharm. Biopharm. 105, 122-133. https://doi.org/10.1016/j.ejpb.2016.06.007

Belkheeva, R.K., 2015. Equation of state for a highly porous material. High Temp. 53, 348357. https://doi.org/10.1134/S0018151X15020054

Çelik, M., 1992. Overview of Compaction Data Analysis Techniques. Drug Dev. Ind. Pharm. 18, 767-810. https://doi.org/10.3109/03639049209058560

Çelik, M., Marshall, K., 1989. Use of a Compaction Simulator System in Tabletting Research. Drug Dev. Ind. Pharm. 15, 759-800. https://doi.org/10.3109/03639048909058530

Chakraborty, M., Bawuah, P., Tan, N., Ervasti, T., Pääkkönen, P., Zeitler, J.A., Ketolainen, J., Peiponen, K.-E., 2016. On the correlation of effective terahertz refractive index and average surface roughness of pharmaceutical tablets. J. Infrared, Millimeter, Terahertz Waves 37, 776-785. https://doi.org/10.1007/s10762-016-0262-0

Chakraborty, M., Ridgway, C., Bawuah, P., Markl, D., Gane, P.A.C., Ketolainen, J., Zeitler, J.A., Peiponen, K.-E., 2017. Optics-based compressibility parameter for pharmaceutical tablets obtained with the aid of the terahertz refractive index. Int. J. Pharm. 525, 85-91. https://doi.org/10.1016/j.ijpharm.2017.03.093

Cooper, A.R., Eaton, L.E., 1962. Compaction Behavior of Several Ceramic Powders. J. Am. Ceram. Soc. 45, 97-101. https://doi.org/10.1111/j.1151-2916.1962.tb11092.x

European Medicines Agency, 2012. Guideline on Real Time Release Testing. 
Han, L.H., Elliott, J.A., Bentham, A.C., Mills, A., Amidon, G.E., Hancock, B.C., 2008. A modified Drucker-Prager Cap model for die compaction simulation of pharmaceutical powders. Int. J. Solids Struct. 45, 3088-3106. https://doi.org/doi:10.1016/j.ijsolstr.2008.01.024

Heckel, R.W., 1961a. Density-Pressure Relationships in Powder Compaction. Trans. Metall. Soc. AIME 221, 671-675.

Heckel, R.W., 1961b. An Analysis of Powder Compaction Phenomena. Trans. Metall. Soc. AIME 221, 1001-1008.

Hersey, J., Rees, J., 1971. Particles-deformation of particles during briquetting. Nature 230, 96. https://doi.org/10.1038/physci230096a0

Kawakita, K., Lüdde, K.-H., 1971. Some considerations on powder compression equations. Powder Technol. 4, 61-68. https://doi.org/https://doi.org/10.1016/0032-5910(71)800013

Kolařík, J., 1994. A model for the yield strength of binary blends of thermoplastics. Polymer (Guildf). 35, 3631-3637. https://doi.org/10.1016/0032-3861(94)90539-8

Krycer, I., Pope, D.G., Hersey, J.A., 1982. The Interpretation of Powder Compaction Data - a Critical Review. Drug Dev. Ind. Pharm. 8, 307-342. https://doi.org/10.3109/03639048209022103

Kuentz, M., Leuenberger, H., 1999. Pressure susceptibility of polymer tablets as a critical property: A modified Heckel equation. J. Pharm. Sci. 88, 174-179. https://doi.org/10.1021/js980369a

Lee, S.L., O’Connor, T.F., Yang, X., Cruz, C.N., Chatterjee, S., Madurawe, R.D., Moore, C.M.V., Yu, L.X., Woodcock, J., 2015. Modernizing Pharmaceutical Manufacturing: from Batch to Continuous Production. J. Pharm. Innov. https://doi.org/10.1007/s12247$015-9215-8$

Lüdde, K., Kawakita, K., 1966. Die Pulverkompression. Pharmazie 21, 393-403.

Markl, D., Bawuah, P., Ridgway, C., van den Ban, S., Goodwin, D.J., Ketolainen, J., Gane, P., Peiponen, K.-E., Zeitler, J.A., 2017a. Fast and Non-destructive Pore Structure Analysis using Terahertz Time-Domain Spectroscopy. Int. J. Pharm. 537, 102-110. https://doi.org/10.1016/j.ijpharm.2017.12.029 
Markl, D., Strobel, A., Schlossnikl, R., Bøtker, J., Bawuah, P., Ridgway, C., Rantanen, J., Rades, T., Gane, P., Peiponen, K.-E., Zeitler, J.A., 2018a. Characterisation of Pore Structures of Pharmaceutical Tablets: A Review. Int. J. Pharm. 538, 188-214. https://doi.org/https://doi.org/10.1016/j.ijpharm.2018.01.017

Markl, D., Wang, P., Ridgway, C., Karttunen, A.-P., Bawuah, P., Ketolainen, J., Gane, P., Peiponen, K.-E., Zeitler, J.A., 2018b. Resolving the rapid water absorption of porous functionalised calcium carbonate powder compacts by terahertz pulsed imaging. Chem. Eng. Res. Des. 132, 1082-1090. https://doi.org/10.1016/j.cherd.2017.12.048

Markl, D., Wang, P., Ridgway, C., Karttunen, A.-P., Chakraborty, M., Bawuah, P., Pääkkönen, P., Gane, P., Ketolainen, J., Peiponen, K.-E., Zeitler, J.A., $2017 b$. Characterization of the Pore Structure of Functionalized Calcium Carbonate Tablets by Terahertz Time-Domain Spectroscopy and X-Ray Computed Microtomography. J. Pharm. Sci. 106, 1586-1595. https://doi.org/10.1016/j.xphs.2017.02.028

Nasr, M.M., Krumme, M., Matsuda, Y., Trout, B.L., Badman, C., Mascia, S., Cooney, C.L., Jensen, K.D., Florence, A., Johnston, C., Konstantinov, K., Lee, S.L., 2017. Regulatory Perspectives on Continuous Pharmaceutical Manufacturing: Moving From Theory to Practice: September 26-27, 2016, International Symposium on the Continuous Manufacturing of Pharmaceuticals, in: Journal of Pharmaceutical Sciences. Elsevier, pp. 3199-3206. https://doi.org/10.1016/j.xphs.2017.06.015

Paronen, P., 1986. Heckel plots as indicators of elastic properties of pharmaceuticals. Drug Dev. Ind. Pharm. 12, 1903-1912. https://doi.org/10.3109/03639048609042616

Rasenack, N., Müller, B.W., 2002. Crystal habit and tableting behavior. Int. J. Pharm. 244, 45-57. https://doi.org/10.1016/S0378-5173(02)00296-X

Rue, P.J., REES, J.E., 1978. Limitations of the Heckel relation for predicting powder compaction mechanisms. J. Pharm. Pharmacol. 30, 642-643. https://doi.org/10.1111/j.2042-7158.1978.tb13347.x

Ryshkewitch, E., 1953. Compression Strength of Porous Sintered Alumina and Zirconia. J. Am. Ceram. Soc. 36, 65-68. https://doi.org/10.1111/j.1151-2916.1953.tb12837.x

Salleh, F.S.M., Yusof, Y.A., Anuar, M.S., Chin, N.L., 2015. Understanding the tabletting characteristics of Ficus deltoidea powder by fitting into compression models. J. Food 
Stirnimann, T., Atria, S., Schoelkopf, J., Gane, P.A.C., Alles, R., Huwyler, J., Puchkov, M., 2014. Compaction of functionalized calcium carbonate, a porous and crystalline microparticulate material with a lamellar surface. Int. J. Pharm. 466, 266-275. https://doi.org/10.1016/j.ijpharm.2014.03.027

Sun, C., 2004. A Novel Method for Deriving True Density of Pharmaceutical Solids Including Hydrates and Water-Containing Powders. J. Pharm. Sci. 93, 646-653. https://doi.org/10.1002/jps.10595

Sun, C., Grant, D.J.W., 2001. Influence of Elastic Deformation of Particles on Heckel Analysis. Pharm. Dev. Technol. 6, 193-200. https://doi.org/10.1081/PDT-100000738

Van Veen, B., Van Der Voort Maarschalk, K., Bolhuis, G.K., Frijlink, H.W., 2004. Predicting mechanical properties of compacts containing two components. Powder Technol. 139, 156-164. https://doi.org/10.1016/j.powtec.2003.11.003

Wax, R.M., Cleek, G.W., 1973. The Effect of Temperature and Pressure on the Refractive Index of Some Oxide Glasses. J. Res. Natl. Bur. Stand. - A. Phys. Chem. 77A, 755-763. https://doi.org/10.6028/jres.077A.046

Wu, C.Y., Best, S.M., Bentham, A.C., Hancock, B.C., Bonfield, W., 2005. A simple predictive model for the tensile strength of binary tablets. Eur. J. Pharm. Sci. 25, 331336. https://doi.org/10.1016/j.ejps.2005.03.004

Wurster, D.E., Buckner, I.S., 2012. Characterizing compaction-induced thermodynamic changes in a common pharmaceutical excipient. J. Pharm. Sci. 101, 2960-2967. https://doi.org/https://doi.org/10.1016/j.ijpharm.2018.01.017

\section{Table 1}

\begin{tabular}{ccccc}
\hline Tablet batch & $m(\mathrm{mg})$ & $H_{\text {out }}(\mathrm{mm})$ & $D(\mathrm{~mm})$ & $f_{\text {out }}(\%)$ \\
\hline B01 & $217 \pm 2$ & 1.67 & 10.04 & $45.50 \pm 0.52$ \\
B02 & $190 \pm 2$ & 1.64 & 10.03 & $50.27 \pm 0.50$ \\
B03 & $168 \pm 2$ & 1.63 & 10.02 & $55.72 \pm 0.40$
\end{tabular}




$\begin{array}{lllll}\text { B04 } & 147 \pm 2 & 1.62 & 10.02 & 61.02 \pm 0.93 \\ \text { B05 } & 122 \pm 2 & 1.61 & 10.00 & 67.35 \pm 0.79\end{array}$

528 Table 1: A list of the out-of-die properties of the five batches of FCC tablets. The tablet

529 geometry was that of flattop cylindrical, having an axial height, $H_{\text {out }}$, diameter, $D$, weight, $m$,

530 and the calculated nominal porosity, $f_{\text {out }}$, derived from the true density and bulk density. The

531 instruments used for measurement of weight, dimensions as well as the porosity calculation

532 have been described in our previous study (Markl et al., 2017b).

533 Table 2

\begin{tabular}{ccccc}
\hline Tablet batch & $f_{\text {in }}(\%)$ & $\delta f(\%)$ & $H_{\text {in }}(\mathrm{mm})$ & $\delta H(\%)$ \\
\hline B01 & 44.04 & 3.32 & 1.60 & 4.37 \\
B02 & 49.95 & 0.64 & 1.56 & 5.13 \\
B03 & 55.56 & 0.29 & 1.57 & 3.82 \\
B04 & 60.97 & 0.08 & 1.55 & 4.52 \\
B05 & 67.36 & 0.01 & 1.56 & 3.21
\end{tabular}

534 Table 2: Estimated in-die values of the average porosity and average height of the five batches 535 of FCC compacts. $f_{\text {in }}$ is the in-die porosity values at maximum compressive pressure. $\delta f$ is the 536 relative change, in percentage, between the out-of-die (see Table 1) and in-die porosity values. $537 H_{\text {in }}$ is the in-die tablet height at maximum compressive pressure. $\delta H$ is the relative height 538 change, in percentage, between the out-of-die (see Table 1)and in-die data

$539 \quad$ Table 3

\begin{tabular}{cccccc}
\hline Tablet batch & $n_{\text {eff.out }}$ & $n_{\text {eff.in }}$ & $\delta n_{\text {eff }}(\%)$ & $n_{\text {in }}(0)$ & $\delta n(0)(\%)$ \\
\hline B01 & 2.152 & 2.172 & 0.92 & 3.053 & 2.79 \\
B02 & 2.029 & 2.000 & 1.45 & 3.149 & 6.03 \\
B03 & 1.916 & 1.884 & 1.70 & 3.164 & 6.53 \\
B04 & 1.798 & 1.789 & 0.50 & 3.111 & 4.75 \\
B05 & 1.669 & 1.711 & 2.45 & 2.964 & 0.20
\end{tabular}

540 Table 3: A comparison between measured $\mathrm{THz}$ effective refractive index ( $n_{\mathrm{eff} . o u t}$ ) and

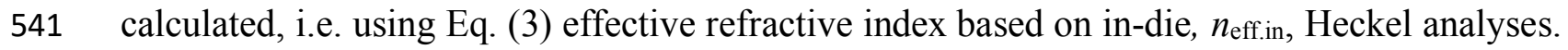
$542 n_{\text {in }}(0)$ is the calculated zero-porosity refractive index using the in-die $\mathrm{K}$ and A values obtained 543 from the conventional Heckel plots (Fig. 2). $\delta n_{\text {eff }}$ is the relative change, in percentage, between 544 the out-of-die and in-die effective refractive index values whereas $\delta n(0)$ is the calculated 
relative change in the intrinsic refractive index of FCC using out-of-die, $n_{\text {out }}(0)=2.97$, and indie, $n_{\text {in }}(0)$ refractive indices. Eq. (3), in conjunction with out-of-die effective refractive index

547 ( $\left.n_{\text {eff.out}}\right)$, was used for the calculation of the in-die zero-porosity refractive indices tabulated 548 below.

549

550

551

552

553

554

555

556

557

558

559

560

561

562

563
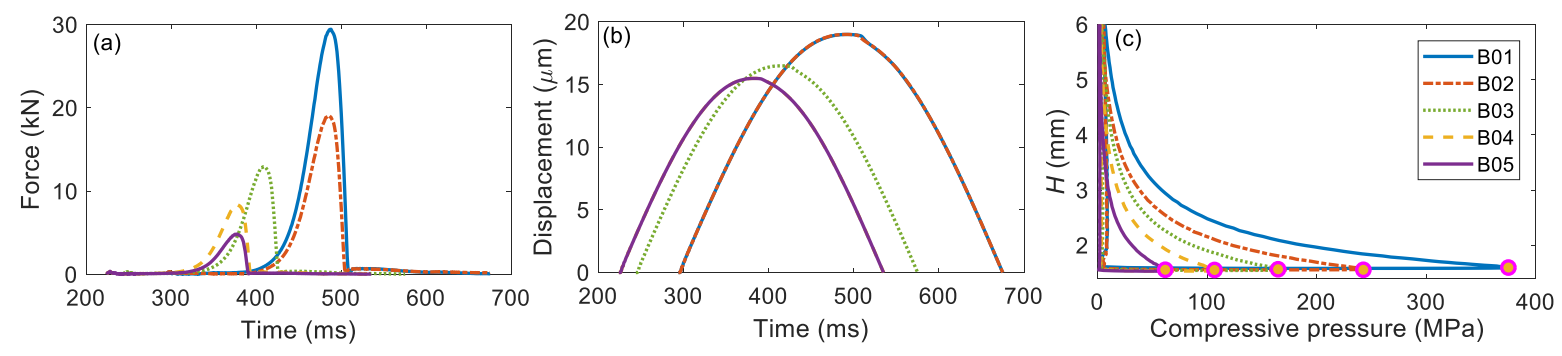

Fig. 1. Parameters of the compaction of five batches with varying porosity. (a) The compressive force- and (b) displacement-time profiles for each batch of tablets during compression. (c) Indie monitoring of the height of the tablets during the compression. The targeted output tablet height was around $1.5 \mathrm{~mm}$.

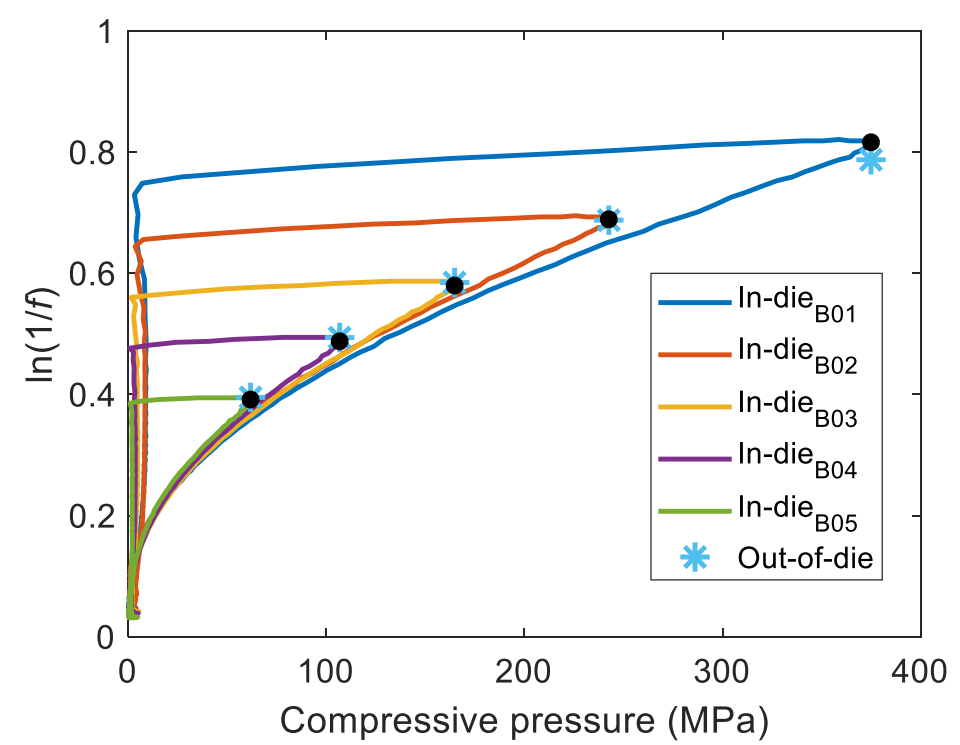

Fig. 2. Conventional Heckel plots for the tablet samples using in-die and out-of-die measurements. From the log-linear extrapolation $\left(R^{2}=0.99\right)$ using batch B01 for the in-die analysis, the values of $K=0.00130 \mathrm{MPa}^{-1}$ and $A=0.3361$ were extracted. Out-of-die analysis of the five batches of FCC tablets using two points for the log-linear extrapolation yielded $K=$ $0.00076 \mathrm{MPa}^{-1}$ and $A=0.5050$. Only batch B01 was used for the in-die analysis due to the well-defined linear portion between the pressure range of around $250-370 \mathrm{MPa}$, as shown.

This pressure range matches the portion chosen for the log-linear fitting for the out-of-die analysis. The black dots show the in-die values of $\ln (1 / f)$ at the maximum compressive pressure for each batch. 


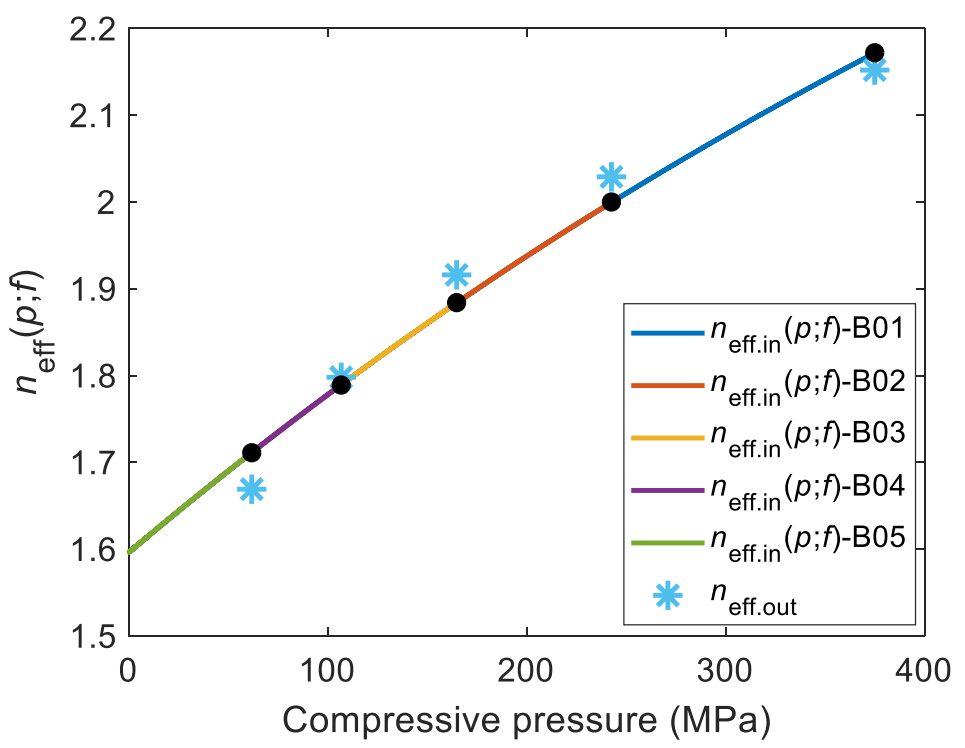

564

Fig. 3. Effective refractive index $\left(n_{\mathrm{eff}}(p ; f)\right)$ as a function of the compaction pressure for both 566 in-die and out-of-die analysis. The parameters $K=0.00130 \mathrm{MPa}^{-1}, A=0.3361$, obtained from 567 the conventional Heckel plots, were used to calculate the $n_{\text {eff.in }}(p ; f)$. For the sake of comparison, 568 we again plot the out-of-die measured $\mathrm{THz}$ refractive index, $n_{\text {eff.out, }}$ as a function of compressive 569 pressure. The black dots show the values of $n_{\text {eff.in }}(p ; f)$ at the maximum compressive pressure 570 for each batch.

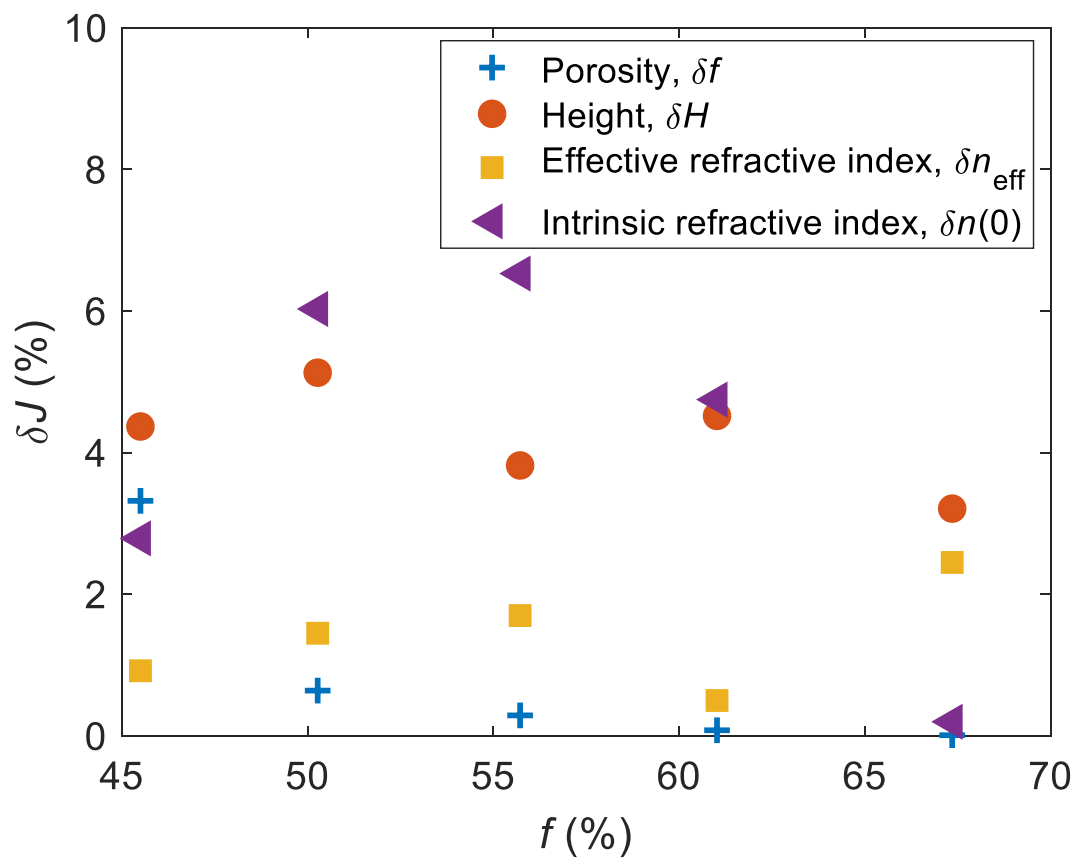

571

572 Fig. 4. A comparison of the estimated relative change in the porosity, height, effective 573 refractive index and intrinsic refractive index with respect to the out-of-die porosity. 

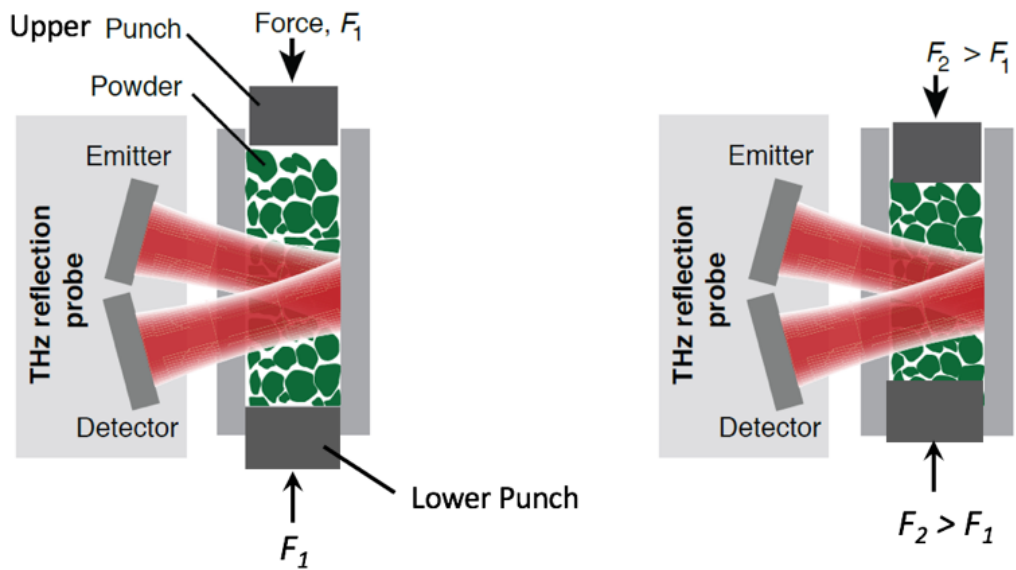

575 Fig. 5. Principle of a proposed future compaction setup coupled with a TPI. (a) Shows the very 576 beginning of the compression process with the force, $F_{1}$. (b) Indicates an increasing 577 compressive force, $F_{2}$, which causes an increase of the refractive index. This designed setup 578 will monitor the dynamic change of the $\mathrm{THz}$ refractive index from the beginning to the end of 579 the compaction process. 